\title{
Potential Neurogenesis and Neuroprotective Effects of Epigallocatechin-3-gallate (EGCG) in Green Tea (Camellia sinensis) Through Microglia M2 Induction Process and NLRP3 Inhibition as an Innovation for Ischemic Stroke Adjuvant Therapy: A Review
}

\author{
Innas Safira Putri ${ }^{1}$, Tazkia Nafs Robbani ${ }^{1}$, Dinda Divamillenia ${ }^{1}$, Octaviana Galuh \\ Pratiwi ${ }^{1}$, Reny I'tishom ${ }^{2, *}$ \\ Email: ritishom@fk.unair.ac.id \\ ${ }^{1}$ Medical Program, Faculty of Medicine Universitas Airlangga, 60132, Surabaya, East Java, Indonesia \\ ${ }^{2}$ Department of Biomedical Science, Faculty of Medicine Universitas Airlangga, 60132, Surabaya, East Java, Indonesia
}

\begin{abstract}
Stroke is a condition that occurs when blood vessels in the brain become obstructed or rupture, cutting off blood flow to the brain and causing brain cells to die. Stroke is divided into two types based on the cause: ischemic stroke and hemorrhagic stroke. Ischemic stroke is the most common kind of stroke, which accounts for approximately $87 \%$ of all strokes. The high prevalence of ischemic stroke has far-reaching consequences for society. Because ischemic stroke is still common and treatment options are limited, a therapeutic breakthrough that can help reduce stroke mortality and morbidity, particularly ischemic stroke, is required. One of the advancements that can be made is the consumption of green tea (Camellia sinensis), which has neurogenetic and neuroprotective benefits. This literature was compiled through online searches engines using Pubmed, Sciencedirect, NIH NCBI, and Google Scholar instruments. In this way, green tea with the active ingredient Epigallocatechin-3-gallate (EGCG) has the potential to induce neurogenesis in ischemic conditions by causing microglia to polarize to $\mathrm{M} 2$ and can inhibit pro-inflammatory mediators by inhibiting NOD-like Receptor Protein-3 (NLRP3), thereby inhibiting the pyroptosis process. As a result, EGCG has the potential to become a neuroprotective ingredient. These two major processes will aid in the recovery of ischemic stroke patients.
\end{abstract}

Keywords: EGCG, Green Tea, Camellia sinensis, M2 Microglia, NLRP3, Ischemic Stroke, Neurogenesis, Neuroprotective

\section{Introduction}

Stroke is a disease of the blood vessels in the brain, specifically the condition of blood vessel blockage or rupture, which cuts off blood flow to the brain and causes cell death in some areas of the brain [1]. Because cell death in the brain results in the loss of neurological function, stroke is one of the leading causes of death and disability worldwide. It is worth noting that stroke is the world's second leading cause of death and third leading cause of disability [2]. Stroke is classified into two types based on its etiology: ischemic stroke and hemorrhagic stroke. Ischemic stroke got the first rank as the most common type of stroke (87\%) [3] caused by cerebral artery occlusion [1].

The high prevalence of ischemic stroke has an impact on the community, one of which is a high economic burden, both in terms of treatment and due to the patient's disability. Until now, the Food and Drug Administration has approved only the rtPA (Alteplase) class of thrombolytic therapy for use in stroke patients, but the safety and efficacy of this therapy are still limited [4]. Because of the high prevalence and limited treatment options, it is critical to develop a therapeutic innovation that will improve the well-being 
of stroke mortality and morbidity, particularly ischemic stroke. The purpose of this literature review is to determine the potential of EGCG in green tea as an adjuvant therapy candidate and to play a role in the prevention of ischemic stroke by inducing M2 microglia and inhibiting NLRP3.

\section{Methods}

This review method evolved from several related literature studies and is supported by discussion analysis. The literature search was conducted using an online search method that included the instruments using Pubmed, Sciencedirect, NIH NCBI, and Google Scholar. Meanwhile, keywords such as EGCG, Green Tea, Camellia sinensis, M2 Microglia, NLRP3, Ischemic Stroke, Neurogenesis, and Neuroprotective have been used. Limitations of English and Indonesian publications in the last ten years revealed 49 types of literature from scan results, including systematic reviews, meta-analysis, randomized controlled trials, case-control studies, and literature in other forms such as dissertations.

\section{Results and Discussion}

\subsection{The Role of M2 Microglia in Neurogenesis}

The ischemic stroke will initiate several processes that will result in tissue damage [5]. Peripheral microglia and macrophages will rapidly arrive at the site of tissue damage in the brain, releasing effector molecules and recruiting other immune cells [6]. The main glial components of the central nervous system (CNS) are known as microglia. Microglia function as tissue-dwelling macrophages that are very sedentary without significant changes from outside the CNS, but they are very dynamic locally [7]. Within a few hours after ischemia, microglia will respond to environmental changes by changing their morphology and increasing their contact with neurons [8]. Microglia undergo rapid morphological transformations from a resting to an active state and then release a variety of cytokines that aid in cell damage or repair. Through different signaling pathways, resting microglia can be classically polarized into an M1 phenotype (involved in tissue injury) or an M2 phenotype (involved in neurogenesis and functional recovery) $[6,9]$.

Polarization of M1 will cause the release of pro-inflammatory cytokines, Nitric Oxide (NO), Reactive Oxygen Intermediates (ROIs), and tissue damage. On the other hand, the polarization of M2 will produce anti-inflammatory cytokines such as Interleukin-10 (IL-10), Transforming Growth Factor Beta (TGF- $\beta$ ), and Arginase-1 (Arg-1) to overcome inflammation, so that this condition can provide an environment that supports neurogenesis as compensation for neuronal repair [10,11,12]. M2 has been known to inhibit inflammation, promote tissue repair and remodeling, and play a role in neurogenesis and functional repair $[9,10,12]$. In an in vivo study published in 2016, Zhang et al. discovered that the microglial response to the M2 phenotype was associated with increased proliferation of Neural Progenitor Cells (NPCs) in the subventricular zone and migration of subventricular neuroblasts. Evidence suggests that microglia polarized to the M2 phenotype can counteract excessive inflammatory activation and promote tissue repair following cerebral ischemia [12]. Microglia play a role in the maturation of newly formed nerve cells as well. As a result, the M2 phenotype has been identified as a key promoter of post-stroke brain repair. The M2 phenotype of microglia can influence neurogenesis by secreting anti-inflammatory factors such as Insulin-like Growth Factor (IGF) and Brain-Derived Neurotrophic Factor (BDNF) [13].

\subsection{The Role of EGCG in Inducing Microglial M2 Elevation}

Microglia that are initially at rest can be polarized into one of two phenotypes: M1 or M2 [9]. M1 produces pro-inflammatory cytokines as well as NO and ROIs, which cause tissue damage. When anti- 
inflammatory cytokines such as IL-4, IL-13, and IL-10 are stimulated, M2 will polarize via alternative pathways $[10,11]$. The administration of EGCG was able to increase M2 polarization while inhibiting M1 polarization. EGCG can prevent the formation of NO, a compound that causes oxidative stress $[10,14]$. Furthermore, EGCG has been shown to downregulate inducible Nitric Oxide Synthase (iNOS) and Cyclooxygenase-2 (COX-2) gene expression, preventing the production of pro-inflammatory mediators such as NO and Prostaglandin E2 (PGE2), as well as EGCG can strongly inhibit leukocyte elastase, which mediates the activation of inflammation inducers such as matrix metalloproteinase-2 (MMP-2) and MMP-9. In other words, EGCG acts as an anti-inflammatory agent. As a result, EGCG accelerates the shift from M1 to M2 [5,10,14]. According to a study published in 2016 by Zhang et al., treatment of EGCG during stroke recovery is associated with elevated microglial responses in the form of polarization of the M2 phenotype [12].

Activated M2 upregulates scavenger receptors such as the mannose receptor (CD206) on its cell surface $[10,11]$. CD206 is regarded as a particularly reliable M2 indicator or marker because it is frequently expressed during M2 activation but not in the M1 phenotype [15,16]. CD206 can remove inflammatory chemicals from the circulation, which aids in inflammation resolution [17]. CD206 expression as an M2 marker increases dramatically when M2 is activated by IL-4, IL-13, and IL-10. CD206 expression, on the other hand, decreases when M2 is induced by (IFN $\gamma$ ) and Lipopolysaccharide (LPS) [16,18]. EGCG can inhibit the induction of IFN and LPS regulation while increasing IL-10 and IL-13. Finally, EGCG can aid in the rise of CD206 expression in M2 $[12,19,20,21]$.

\subsection{The Role of NLRP3 in the Pyroptosis Process}

NLRP3 is a key mediator of the innate immune response to danger signals as well as a proinflammatory factor [22]. When activated, NLRP3 causes the pyrine domain of the NLR to bind to an adaptor protein called Apoptosis-associated Spec-like protein containing CARD (ASC). ASC will trigger numerous NLRP3 monomers to bind to each other via the oligomerization process, resulting in the formation of the NLRP3 inflammasome, whose primary role is to convert pro-caspase1 to caspase1 [23]. Caspase-1 cleaves pro-IL-1 and pro-IL-18 into more mature forms (IL-1 and IL-18), as well as gasdermin D (GSDMD) to gasdermin N, which causes pore opening and pyroptosis to induce pore opening along with pyroptosis [24]. Pyroptosis is essential in the development of ischemic stroke [25].

The mechanism of pyroptosis in microglia by NLRP3 during ischemic stroke is induced by several pathways, including HMGB1, loss of ion homeostasis, and mitochondrial damage.

\section{- HMGB1}

During ischemia conditions, High Mobility Group Box Protein 1 (HMGB1), a DamageAssociated Molecular Pattern Molecule (DAMP), is released from the damaged neurovascular unit and dies along with other DAMPs during ischemia. HMGB1 binds to Toll-like Receptor 2 (TLR2), TLR4, and Receptor for Advanced Glycation End-Products (RAGE) on microglia cells, activating the transcription factor Nuclear Factor-kB (NF- $\mathrm{kB})$. The NF- $\mathrm{kB}$ and Adenosine Triphosphate (ATP) are required for NLRP3 activation [26].

\section{- Ionic Dishomeostasis}

During the acute phase of a stroke, blood flow will decrease, causing the amount of ATP to decrease and lactic acidosis. Brain tissue is oxygen depleted. This will result in a loss of $\mathrm{K}+$ and $\mathrm{Ca} 2+$ ion balance in microglia cells. The homeostasis of these ions affects mitochondrial damage. Mitochondria are the primary modulators of the NLR family of NLRP3 inflammasome activation [27]. The influx of $\mathrm{Ca} 2+$ indirectly contributes to NLRP3 activation. Meanwhile, intracellular K+ 
levels that are lower than the threshold will also activate NLRP3 [28].

\section{- Mitochondrial Damage}

Mitochondria are the primary modulators of NLRP3 inflammasome activation in the NLR family [27]. The outer mitochondrial membrane serves as a platform for inflammasome assembly, activation of innate immune responses, and pyroptosis processes via various pro-inflammatory cytokines and caspase-1 [29].

Mitochondrial function damage causes excessive production of Reactive Oxygen Species (ROS). This ROS generation was identified as the first to trigger NLRP3 activation [30]. Evidence supports a correlation between ROS overproduction and neuronal death in a variety of neurological disorders, including amyotrophic lateral sclerosis, epilepsy, alzheimer's disease, parkinson's disease, ischemic stroke, and traumatic brain injury. Excessive ROS levels cause functional and structural changes in brain tissue and play an important role in the pathogenesis of cerebral ischemia. As a result, mitochondrial dysfunction and high oxidative stress are significant contributors to the ongoing ischemia process.

\subsection{The Potential of EGCG in Pyroptosis Inhibition}

Bitu et al. (2015) discovered that green tea extracts and catechins (EGCG) had a neuroprotective effect in a mouse striatal PD 6-OHDA model (the neurotoxin 6-hydroxydopamine, which is commonly used to create Parkinson's disease models) [31]. Meanwhile, in a study conducted by Machin (2020), green tea with the active ingredient EGCG was shown to inhibit neuronal cell death via the pyroptosis process in the Rattus norvegicus Middle Cerebral Artery Occlusion (MCAO) model [32]. This study also discovered that the active ingredient EGCG, as well as green tea extract, have an anti-necroptosis effect due to their high antioxidant content [33]. Green tea contains the antioxidant EGCG as an active component. EGCG promotes HMGB1 degradation by conjugating with its cytoplasm to create the EGCG-HMGB1 complex. Because of its large size, this complex cannot physically pass through the ubiquitin-proteasome groove's narrow proteasome pore. So, it starts the autophagic process [34].

EGCG can also restore $\mathrm{Ca} 2+$ homeostasis through downregulation of the protein kinase RNA-like endoplasmic reticulum kinase-activating transcription factor 6 (PERK-ATF4) signaling pathway, Inositol-requiring enzyme-1a (IRE1a), and activating transcription factor 6 (ATF6) [35]. In some studies, it is also known that EGCG can inhibit ROS production [36,37]. Some of these ROS inhibition mechanisms will then inhibit NLRP3 until the process described in the preceding paragraph occurs.

Furthermore, EGCG is a mitochondrion-targeting medicinal drug that controls mitochondrial metabolism, including mitochondrial biogenesis, bioenergetics, and mitochondrial-mediated cell cycle and apoptosis. Mitochondrial biogenesis occurs as a result of EGCG-induced activation of Peroxisome proliferator-activated receptor gamma coactivator 1-alpha (PGC-1a) [38]. 


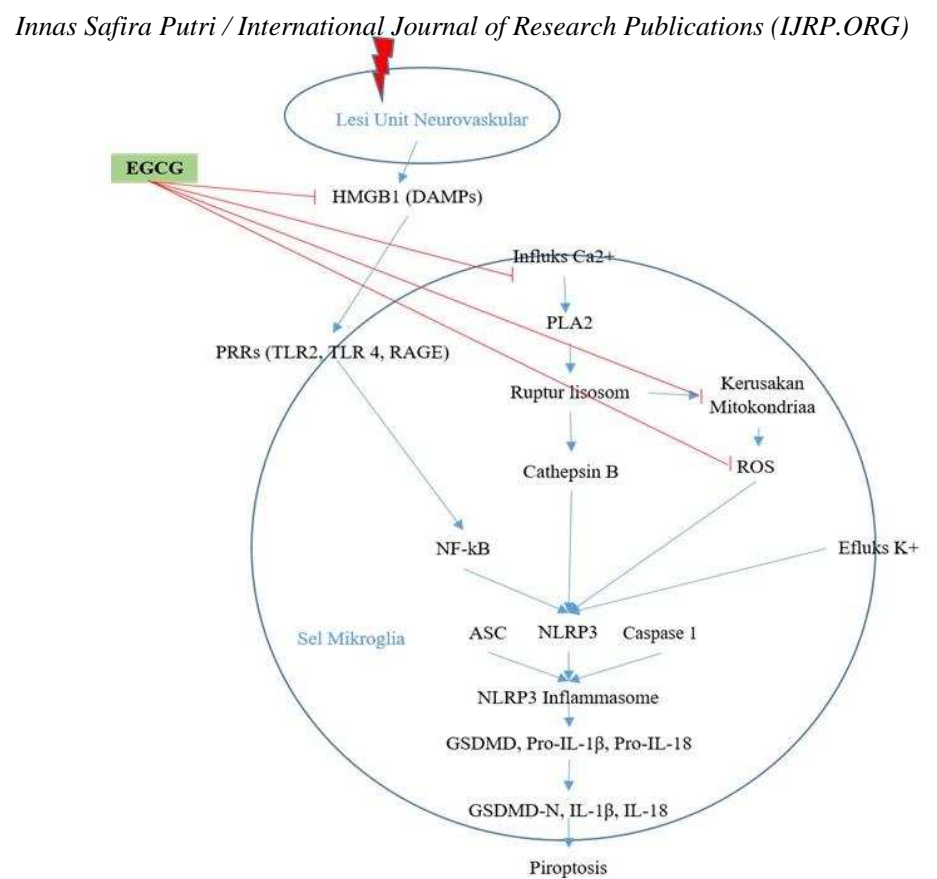

Fig. 1. Mechanism of NLRP3 Inhibition in Multiple Pathways by EGCG in Microglia Cells

\subsection{Neurogenesis and Neuroprotective Effects of EGCG in The Green Tea (Camellia sinensis) as Ischemic Stroke Prevention and Adjuvant Therapy}

Green tea is a well-known beverage around the world, and consuming green tea is thought to have health benefits [39]. Moreover, green tea has gained popularity due to its polyphenol content, which can act as antioxidants, free radical scavengers, and protect against neurodegeneration, inflammatory disorders, and cancer. here are several methods for producing different types of tea, such as black tea (completely oxidized), green tea (not oxidized), and oolong tea (partially oxidized) [40]. Both black and green tea are high in antioxidants, which may help reduce the risk of stroke and have a preventive effect, but green tea is more widely consumed [41].

Green tea has been shown to increase antioxidant activity as well as total polyphenols when consumed at a rate of two to three cups per day for a lengthy period [32,33]. Several other studies have found that drinking up to three cups of green tea per day can reduce the incidence of overall stroke and ischemic stroke by $13 \%$ and $24 \%$, respectively [42]. Green tea contains four major polyphenols (commonly known as catechins): EGCG, epicatechin gallate (ECG), epigallocatechin (EG), and epicatechin (EC) [33,43]. Green tea is high in EGCG polyphenols, with brewed teacups containing 60 65 percent EGCG from $240-320 \mathrm{mg}$ catechins and 20-50 mg caffeine [44,45]. Green tea's EGCG has piqued the interest of researchers due to its ability to cross the blood-brain barrier and reach the brain parenchyma [46,47].

Green tea contains EGCG, which is thought to have neurogenic and neuroprotective effects on tissues through a variety of mechanisms. For example, giving EGCG to tissues experiencing ischemiareperfusion causes a decrease in the expansion of ischemia [32] and can prevent cerebral ischemic injury as well as improve changes in morphogenesis in the brain [47,48]. This is supported by the mechanism of inhibiting NO formation as a compound that causes oxidative stress [10,14], downregulating inducible iNOS and COX-2 gene expression, and strongly inhibiting leukocyte elastase, which mediates the 
activation of MMP-2 and MMP-9 [5,10,14], which inhibit expression that induces IFN and LPS regulation and increases IL-10 and IL13 [12,19,20,49]. From some of these mechanisms, the administration of EGCG can prevent the production of pro-inflammatory mediators. In other words, EGCG has anti-inflammatory properties. Under these conditions, EGCG has the ability to promote M2 polarization while inhibiting M1 polarization in brain tissue. Furthermore, M2 will release antiinflammatory cytokines to combat the existing inflammation, allowing compensatory neural repair in the form of a neurogenesis process to occur [12]. This is supported by evidence from a study conducted by Zhang et al. (2016), which discovered that administering EGCG during stroke recovery significantly improves functional recovery by increasing NPC proliferation in the subventricular zone and subventricular neuroblast migration, which could be associated with microglial responses in the form of M2 phenotype polarization [12].

Aside from its effect on neurogenesis, EGCG has been shown to suppress neuronal cell death via pyroptosis in the Rattus norvegicus MCAO model [31]. EGCG can induce HMGB1 breakdown, restore $\mathrm{Ca} 2+$ homeostasis, and act as a mitochondrion-targeting therapeutic drug. Furthermore, EGCG has been shown to reduce ROS production [37,47]. All of these processes will have an effect on NLRP3 inhibition, causing the pyroptosis process to be inhibited as well. Based on the numerous mechanisms provided by EGCG, it may be a solution for use as adjuvant therapy in patients with ischemic stroke. Additionally, Wang et al. founding 2017 support the ability of EGCG in stroke treatment options. In their research [50], they stated that administration of EGCG significantly enhanced treatment outputs of patients in the delayed onset-to-treatment time strata, as made evident by improved the National Institutes of Health Stroke Scale (NIHSS) scores. In their research in the form of a Randomized DoubleBlind and Placebo-Controlled Trial showed that the reduction in plasma levels of both MMP-2 and MMP-9 was likely involved for the better treatment result, as evidenced by significant linear correlations between both MMPs and NIHSS scores in all patients [50]. This further supports the great benefits of EGCG for stroke treatment in the future.

\section{Conclusion}

Green tea (Camellia sinensis) contains EGCG, which is effective as an adjuvant therapy for ischemic stroke because it has a neurogenesis effect via its anti-inflammatory properties, which may also increase M2 polarization and is neuroprotective via the mechanism of inhibiting the pyroptosis process. In the early stages of recovery, the two main processes will help ischemic stroke patients. Additional experimental and clinical trials will be required in the future to obtain more genuine scientific evidence.

\section{Acknowledgements}

The author would like to thank all those who helped in writing this piece of literature.

\section{References}

[1] Kementerian Kesehatan Republik Indonesia. Apa Itu Stroke? - Direktorat P2PTM. 2018. [online] Available at: <http://p2ptm.kemkes.go.id/infographic-p2ptm/stroke/apa-itu-

stroke\#: :text=Definisi\%20menurut\%20WHO\%2C\%20Stroke\%20adalah,lain\%20yang

$\% 20$ jelas\%20selain\%20vascular.> [Accessed 20 October 2020].

[2] WHO. Ischaemic and Haemorrhagic Stroke. 2012. Available at:

<https://www.who.int/medicines/areas/priority_medicines/BP6_6Stroke.pdf?ua=1\#: :t

ext=Stroke\%20is\%20classified\%20on\%20the,stroke\%E2\%80\%9D\%2C\%20(25\%25)\% 5D.> [Accessed 20 October 2020 ].

[3] Mozaffarian, D., Benjamin, E., Go, A., Arnett, D., Blaha, M., Cushman, M., et al. Heart Disease and Stroke Statistics22016 
Update. Circulation. 2016;133.

[4] Schwamm, L., Ali, S., Reeves, M., Smith, E., Saver, J., Messe, S., et al. $\mu$ Temporal Trends in Patient Characteristics and Treatment With Intravenous Thrombolysis Among Acute Ischemic Stroke Patients at GetWith the Guidelines \pm Stroke Hospitals. Circ Cardiovasc Qual Outcomes. 2013;6, p. 543-549.

[5] Singh, N., Mandal, A., Khan, Z. Potential Neuroprotective Properties of Epigallocatechin-3- gallate (EGCG). Nutr J. 2016;15(1).

[6] Hu, X., Li, P., Guo, Y., Wang, H., Leak, R., Chen, S., et al. Microglia/Macrophage Polarization Dynamics Reveal Novel Mechanism of Injury Expansion After Focal Cerebral Ischemia.Stroke. 2012;43(11), p. 3063-3070.

[7] Stoll, G., Jander, S. Microglia 论. Reference Module in Neuroscience and Biobehavioral Psychology, in "Stein J. Reference Module in Neuroscience and Biobehavioral Psychology”. Elsevier. 2016.

[8] Szalay, G., Martinecz, B., Lénárt, N., Környei, Z., Orsolits, B., Judák, L., et al. Microglia Protectagainst Brain Injury and Their Selective Elimination Dysregulates Neuronal Network Activity after Stroke. Nat Commun. 2016;7(1).

[9] Choi, J., Kim, J., Kim, J., Park, J., Lee, W., Lee, J. M2 Phenotype Microglia-derived Cytokine Stimulates Proliferation and Neuronal Differentiation of Endogenous Stem Cells in Ischemic Brain. Exp Neurobiol. 2017;26(1), p. 33-41.

[10] Saqib, U., Sarkar, S., Suk, K., Mohammad, O., Baig, M., Savai, R. Phytochemicals as Modulators of M1-M2 Macrophages in Inflammation. Oncotarget. 2018;9(25), p. 17937-17950.

[11] Scodeller, P., Simón-Gracia, L., Kopanchuk, S., Tobi, A., Kilk, K., Säälik, P., et al. Precision Targeting of Tumor Macrophages with a CD206 Binding Peptide. Sci Rep. 2017;7(1).

[12] Zhang, J., Xu, H., Yuan, Y., Chen, J., Zhang, Y., Lin, Y., et al. Delayed Treatment with Green Tea Polyphenol EGCG Promotes Neurogenesis After Ischemic Stroke in Adult Mice. Mol Neurobiol. 2016;54(5), p. 3652-3664.

[13] Wang, X., Xuan, W., Zhu, Z., Li, Y., Zhu, H., Zhu, L., et al. The Evolving Role of Neuro-immuneInteraction in Brain Repair after Cerebral Ischemic Stroke. CNS Neurosci Ther. 2018;24(12), p. 1100-1114.

[14] Zhong, Y., Chiou, Y., Pan, M., and Shahidi, F. Anti-inflammatory Activity of Lipophilic Epigallocatechin Gallate (EGCG) Derivatives in LPS-stimulated Murine Macrophages. Food Chem. 2012;134, p.742-748.

[15] Xu, Z., Gu, Y., Wang, C., Jin, Y., Wen, X., Ma, J., et al. The M2 Macrophage Marker CD206: a Novel Prognostic Indicator for Acute Myeloid Leukemia. Oncoimmunology. 2019;9(1), p. 1683347.

[16] Wang, S., Cao, M., Xu, S., Shi, J., Mau, X., Yao, X., et al. Luteolin Alters Macrophage Polarization to Inhibit Inflammation. Inflammation. 2019;43(1), p. 95-108.

[17] Rőszer, T. Understanding the Mysterious M2 Macrophage through Activation Markers and Effector Mechanisms. Mediators Inflamm. 2015;2015, p. 1-16.

[18] Martinez-Pomares, L. The Mannose Receptor. J Leukoc Biol. 2012;92(6), p. 1177-1186.

[19] Hagiwara, M., Matsushita, K. Epigallocatechin Gallate Suppresses LPS Endocytosis and Nitric Oxide Productionby Reducing Rab5-caveolin-1 Interaction. Biomed Res. 2014;35(2), p. 145-151.

[20] Giunta, B. The Role of HIV-1 Proteins in Alzheimer's Disease Pathology [Ph.D]. University of South Florida. 2011.

[21] Bao, J., Liu, W., Zhou, H., Gui, Y., Yang, Y., Wu, M., et al. Epigallocatechin-3-gallate AlleviatesCognitive Deficits in APP/PS1 Mice. Curr Med Sci. 2020;40(1), p. 18-27.

[22] Tarallo, V., Hirano, Y., Gelfand, B., Dridi, S., Kerur, N., Kim, Y., et al. DICER1 Loss and Alu RNA Induce Age-Related Macular Degeneration via the NLRP3 Inflammasome and MyD88. Cell. 2012;149(4), p. 847-859.

[23] Shi, J., Zhao, Y., Wang, K., Shi, X., Wang, Y., Huang, H., et al. Cleavage of GSDMD by Inflammatory Caspases Determines Pyroptotic Cell Death. Nature. 2015;526(7575), p. 660- 665.

[24] Jorgensen, I., Rayamajhi, M., Miao, E. Programmed Cell Death as a Defence Against infection. Nat Rev Immunol. 2017;17(3), p. 151-164.

[25] Kesavardhana, S., Kanneganti, T. Mechanisms Governing Inflammasome Activation, Assembly and Pyroptosis Induction. Int Immunol. 2017;29(5), p. 201-210.

[26] Allam, R., Lawlor, K., Yu, E., Mildenhall, A., Moujalled, D., Lewis, R., et al. Mitochondrial Apoptosis is Dispensable for NLRP 3 Inflammasome Activation but Non-apoptotic Caspase-8 is Required for Inflammasome Priming. EMBO rep. 2014;15(9), p. 982-990.

[27] Barone-Rochette, G., Vautrin, E., Rodiere, M., Broisat, A., Vanzetto, G. First Magnetic Resonance Coronary Artery Imaging of Bioresorbable Vascular Scaffold in-Patient. Eur Heart J Cardiovasc Imaging. 2014;16(2), p. 229-229.

[28] Muñoz-Planillo, R., Kuffa, P., Martínez-Colón, G., Smith, B., Rajendiran, T., Núñez, G. K+ Efflux Is the Common Trigger of NLRP3 Inflammasome Activation by Bacterial Toxinsand Particulate Matter. Immunity. 2013;38(6), p. 1142-1153.

[29] Szeto, H., Liu, S., Soong, Y., Alam, N., Prusky, G., Seshan, S. Protection of Mitochondria Prevents High-fat Diet-induced Glomerulopathy and Proximal Tubular Injury. Kidney Int. 2016;90(5), p. 997-1011.

[30] Zhou, R., Yazdi, A., Menu, P., Tschopp, J. Erratum: A Role for Mitochondria in NLRP3 Inflammasome Activation. Nature. 2011;475(7354), p. 122-122.

[31] Bitu Pinto, N., da Silva, Alexandre, B., Neves, K., Silva, A., Leal, L., et al. Neuroprotective Properties of the Standardized Extract fromCamellia sinensis (Green Tea) and Its Main Bioactive Components, Epicatechin and Epigallocatechin Gallate, in 
the 6-OHDA Modelof Parkinson's Disease. Evid Based Complement Alternat Med. 2015;2015, p. 1-12.

[32] Machin, A. Penghambatan Proses Kematian Sel Neuron menggunakan Camillia Sinensis dengan Bahan Aktif EGCG pada RattusNorvegicus Model Stroke Iskemik Akut [Doktor]. Universitas Airlangga. 2020.

[33] Machin, A., Purwanto, D., Nasronuddin, Sugianto, P., Aulanni'am, A., Subadi, I., et al. Camellia sinensis with its Active Compound EGCG can Decrease Necroptosis via Inhibition of HO-1 Expression. Eurasia J Biosci. 2020;14, p. 1813-1820.

[34] Li, W., Zhu, S., Li, J., Assa, A., Jundoria, A., Xu, J., et al. EGCG Stimulates Autophagy and Reduces Cytoplasmic HMGB1 Levels in Endotoxin-stimulated Macrophages. Biochem Pharmacol. 2011;81(9), p. 1152-1163.

[35] Karthikeyan, B., Harini, L., Krishnakumar, V., Kannan, V., Sundar, K., Kathiresan, T. Insights on the Involvement of (-)Epigallocatechin Gallate in ER Stress-mediated Apoptosis in Age-related Macular Degeneration. Apoptosis. 2016;22(1), p. $72-85$.

[36] Wang, D., Wang, Y., Xu, S., Wang, F., Wang, B., Han, K., et al. Epigallocatechin-3-gallate Protects against Hydrogen Peroxide-Induced Inhibition of Osteogenic Differentiation of Human Bone Marrow-Derived Mesenchymal Stem Cells. Stem Cells Int. 2016;2016, p. 1-10.

[37] Ning, J., Ding, D., Song, Y., Zhang, Z., Luo, X., Wan, X. Chemical Constituents Analysis of White Tea of Different Qualities and Different Storage Times. Eur Food Res Technol. 2016;242(12), p. 2093-2104.

[38] Shi, J., Deng, H., Pan, H., Xu, Y., Zhang, M. Epigallocatechin-3-gallate Attenuates Microcystin-LR Induced Oxidative Stress and Inflammation in Human Umbilical Vein Endothelial Cells. Chemosphere. 2017;168, p. 25-31.

[39] Suzuki, Y., Miyoshi, N., Isemura, M. Health-promoting Effects of Green Tea. Proc Jpn Acad Ser B Phys Biol Sci. 2012;88(3), p. 88-101.

[40] Ahmed, S. Stepp, J.R. Pu-erh Tea: Botany, Production, and Chemistry, in "Tea in Health and Disease Prevention". Elsevier Science and Technology, Amsterdam. 2013, p. 59-71.

[41] United Nations. TEA - An INFOCOMM Commodity Profile. United Nations Conf TradeDev. 2016. [Internet], p. 1-25.

[42] Shen, L., Song, L., Ma, H., Jin, C., Wang, J., Xiang, M. Tea Consumption and Risk of Stroke: a Dose-response Metaanalysis of Prospective Studies. J Zhejiang Univ Sci B. 2012;13(8), p. 652-662.

[43] Yu, J., Jia, Y., Guo, Y., Chang, G., Duan, W., Sun, M., et al. Epigallocatechin-3-gallate Protects Motor Neurons and Regulates Glutamate Level. FEBS Lett. 2010;584(13), p. 2921-2925.

[44] Itoh, T., Imano, M., Nishida, S., Tsubaki, M., Mizuguchi, N., Hashimoto, S., et al. (-)- Epigallocatechin-3-gallate Increases The Number of Neural Stem Cells around The Damaged Area after Rat Traumatic Brain Injury. J Neural Transm. 2012;119(8), p. $877-890$.

[45] Habtemariam, S. The Chemical and Pharmacological Basis of Tea (Camellia sinensis (L.) Kuntze) as Potential Therapy for Type 2 Diabetes and Metabolic Syndrome, in:"Medicinal Foods as Potential Therapies for Type-2 Diabetes and Associated Diseases". S. 1st ed. Academic Press, London. 2019.

[46] Ortiz-López, L., Márquez-Valadez, B., Gómez-Sánchez, A., Silva-Lucero, M., Torres-Pérez, M., Téllez-Ballesteros, R., et al. Green Tea Compound Epigallo-catechin-3-gallate (EGCG) Increases Neuronal Survival in Adult Hippocampal Neurogenesis In Vivo and In Vitro. Neuroscience. 2016;322, p. 208-220.

[47] Wang, Y., Li, M., Xu, X., Song, M., Tao, H., Bai, Y. Green Tea Epigallocatechin-3-gallate (EGCG) Promotes Neural Progenitor Cell Proliferation and Sonic Hedgehog Pathway Activation during Adult Hippocampal Neurogenesis. Mol Nutr Food Res. 2012;56(8), p. 1292-1303.

[48] Arab, L., Liu, W., Elashoff, D. $\mu$ Green and Black Tea Consumption and Risk of Stroke. Stroke. 2009;40, p. 1786-1792.

[49] Wu, H., Qi, H., Iwasaki, D., Zhu, B., Shimoishi, Y., Murata, Y., et al. JNK-Dependent NFATc1 Pathway Positively Regulates IL-13 Gene Expression 53 Induced by ( \pm )-epigallocatechin-3-gallate in Human Basophilic KU812 Cells. Free Radic Biol Med. 2009;47, p.1028-1038.

[50] Wang, X.H., You Y.P. Epigallocatechin Gallate Extends Therapeutic Window of Recombinant Tissue Plasminogen Activator Treatment for Brain Ischemic Stroke: A Randomized Double-Blind and Placebo-Controlled Trial. Clin Neuropharmacol. 2017;40(1),p.24-28. 